\title{
Abstracts of oral presentations to the ENT UK meeting, 5 September 2008, London, UK
}

\author{
Model for an ENT clinical undergraduate placement: \\ students' perspectives and objective logbook analysis \\ V Jaiswal, S K Ross*, D Wild $\dagger$, J Whittle $\$$ J McGlashan $\dagger$ † \\ From the Freeman Hospital, Newcastle upon Tyne, the *Raigmore \\ Hospital, Inverness, Scotland, the ${ }^{\dagger}$ Queen's Medical Centre, Not- \\ tingham, and ${ }^{\ddagger}$ Nottingham University, UK
}

\section{Objective}

To gain a better understanding of what fourth year medical students find useful during a formally assessed and highly structured ENT placement arranged through Nottingham University.

\section{Method}

A cross-sectional, qualitative questionnaire study and analysis of contemporaneously completed logbooks, undertaken in December 2006. The study population comprised fourth year undergraduate medical students placed at either Derby Royal Infirmary or the Queen's Medical Centre. The questionnaire was developed to obtain student feedback on the perceived educational experience of out-patient clinics and theatre sessions. The contemporaneous logbook, countersigned by a member of staff at the time of attendance, was analysed for quantitative data, for comparison. Attendance at at least 12 (Queen's Medical Centre) or 14 (Derby Royal Infirmary) sessions was compulsory.

\section{Results}

Seventy-six per cent $(n=128)$ of students returned the questionnaire, and 92 per cent $(n=156)$ of the 168 students' logbooks were analysed (90 students from Queen's Medical Centre and 78 from Derby Royal Infirmary).

Overall, students reported a positive experience in both out-patient clinics and theatre. Surprisingly, students reported an improvement in their skills of history-taking (7 per cent), clinical examination ( 9 per cent) and clinical reasoning (35 per cent), even in a theatre environment. However, one negative finding was that only 39 per cent of students felt that their history-taking skills improved in out-patient clinic sessions. One hundred per cent of students completed the mandatory sessions. Twenty-three per cent $(n=36)$ went to extra clinics and 37 per cent $(n=58)$ went to extra theatre sessions. Ninety-four per cent $(n=146)$ went to at least one other extra, optional session, with 76, 27 and 5 per cent going to two, three and four extra, optional sessions, respectively.

\section{Conclusions}

This qualitative feedback was useful to validate the current venues for medical student teaching. Lack of extra rooms in the out-patients clinic may be one reason why students felt that their history-taking skills had not improved. Student attendance was high, compared with other studies, with many students choosing to do extra sessions.

\section{Assessment of ENT registrars using a virtual reality mastoid surgery simulator}

S McDonald, DJ Alderson, J Powles

From the Torbay Hospital, South Devon, UK

\section{Objective}

To assess the feasibility of using a computerised virtual reality mastoid surgery simulator as an objective measure of competence in mastoid surgery.

\section{Method}

Twenty ENT registrars of varying seniority were recruited to the study. All completed a questionnaire detailing previous mastoid surgery experience, and a self-evaluation of skill level. Participants were then given 15 minutes to practice drilling a virgin temporal bone on the simulator. Following this, 10 minutes were given for the participant to select an appropriately sized burr and to continue a partially completed cortical mastoidectomy. It was explained that credit would be given for a smooth and steady drilling action, avoidance of damage to important structures, and progress made in the task.

A film of the drilling was recorded on the computer, which was later blindly and independently assessed by two consultants. Each assessment involved giving an overall impression score to each participant. In addition, competence in a number of domains (e.g. flow of operation and respect for tissues) was scored in an attempt to introduce greater objectivity, as previously validated in surgical skills research. The domain scores were summated, and the total score used as an alternative to the overall impression score.

Results

The relationship between the scores given by the two consultant observers was investigated using the Spearman rank-order correlation coefficient $(\rho)$. There was a strong positive correlation between the consultants' scores for both the overall impression score $(\rho=0.724, n=20, p<$ $0.0005)$ and the domain total score $(\rho=0.659, n=20$, $p=0.002)$. Spearman correlation coefficients also suggested a strong positive correlation between the overall impression scores and the domain total scores $(\rho=0.863, p<0.0005)$.

There were also strong positive correlations when Spearman rank-order correlation coefficients were used to investigate the relationships between the consultants' scores and both the registrars' year of study and their self-rating (in all cases $\rho>0.5$ and $p<0.05$ ).

\section{Conclusions}

The virtual reality mastoid surgery simulator appeared to be a valid and reliable tool for the assessment of competence and experience in mastoid surgery. It may therefore have a role in the summative assessment of trainees. 


\section{Voice symptom score questionnaire as an audit tool} for routine speech therapy

I Nixon, K MacKenzie, C Sellars, C Dunnett

From the North Glasgow Hospitals NHS Trust, Scotland, UK

\section{Objective}

(1) To assess the voice symptom score questionnaire as an audit tool in routine clinical practice, and (2) to report the effect of voice treatment in an unselected, heterogeneous, dysphonic group as measured by the voice symptom score questionnaire.

\section{Method}

Between 5 August 2003 and 23 October 2006, 515 patient records were prospectively entered into the departmental database. In order to assess the voice symptom score questionnaire, we examined two specific groups from within the database. Group one $(n=75)$ consisted of patients who attended the voice awareness group then attended for a first session of therapy, but who did not complete their individual therapy. These patients received indirect therapy alone. Group two $(n=74)$ attended the voice awareness group and then went on to complete an individual course of voice therapy, so were exposed to both indirect and direct therapies. The Wilcoxon rank sum test and MannWhitney U test were used to calculate statistical significance.

\section{Results}

The median voice symptom score for the group receiving indirect therapy alone was 41 at presentation and 21.5 at discharge $(p<0.01)$. The median voice symptom score for the group receiving both direct and indirect therapy was 41 at presentation and eight at discharge $(p<0.01)$. Comparison of the groups' voice symptom scores at discharge showed a significant difference $(p<0.05)$, with a better outcome for those patients completing both group and individual aspects of therapy.

\section{Conclusions}

Clinician-based perceptual analysis of voice is fundamental to the management of voice disorders. However, such assessment may not fully reflect the impact dysphonia has on a patient's quality of life. Use of a patient-centred, selfreported questionnaire does not replace clinician-based perceptual analysis by a speech therapist, but it can act as an adjunct. We are the first group to publish results for use of the voice symptom score questionnaire in routine clinical practice. Our results suggest that patients who complete a course of therapy which includes both group and individual sessions show greater improvement than those who attend for group therapy alone. Although this study had weaknesses, particularly in relation to patient selection, this is a problem other departments will also face when introducing audit tools to their clinical governance programmes. Our results have helped to set a baseline for future performance in our department, and may also be useful to others as a comparison when assessing patient populations and the efficacy of departmental speech therapy. We found the voice symptom score questionnaire to be a simple and reliable audit tool in the assessment of changes in patients' voice-related quality of life, and we would recommend it to other departments.

Diffusion-weighted versus non-echo planar magnetic resonance imaging in detecting middle-ear cholesteatoma

A Kasbekar, B Kenway, D Scoffings, N Donnelly, P Axon From Addenbrooke's Hospital, Cambridge, UK

\section{Objective}

To compare magnetic resonance (MR) diffusion-weighted imaging and MR non-echo planar imaging in the detection of middle-ear cholesteatoma, within a tertiary referral centre setting which has newly adopted MR non-echo planar imaging.

\section{Method}

We undertook a prospective pilot study of 14 patients with a history of middle-ear cholesteatoma. Patients who were due a 'second look' procedure or who clinically had cholesteatoma were scanned with MR diffusion-weighted imaging and MR non-echo planar imaging sequences. The imaging was reported by a neuro-radiologist blinded to the patient history. At surgery, the presence of cholesteatoma was documented and compared with the preoperative radiology report.

\section{Results}

Of the 14 patients, the diffusion-weighted imaging report did not correlate with the non-echo planar imaging report in 14 per cent $(2 / 14)$ of cases. At surgery, the presence or absence of cholesteatoma correlated with the diffusionweighted imaging report in 79 per cent $(11 / 14)$ of cases, but with the non-echo planar imaging report in only 64 per cent $(9 / 14)$ of cases. This difference was not significant. Our study demonstrated diffusion-weighted imaging to have a sensitivity of 60 per cent and a specificity of 89 per cent. Non-echo planar imaging had a sensitivity of 60 per cent and a specificity of 67 per cent.

\section{Conclusions}

As a result of our pilot study, we conclude that diffusionweighted imaging is at least as accurate if not better than non-echo planar imaging in detecting cholesteatoma of the middle ear. A larger study is currently under way to ascertain whether these differences are significant. There are also other confounding factors, such as ease of image interpretation by the neuro-radiologist when utilising new imaging techniques. Based on the figures above, imaging alone cannot be relied upon to alter decisions on whether to perform a 'second look' procedure or not. Above all, clinical suspicion should be trusted in all cases.

\section{Endoscopic frontal sinus surgery: when is frontal sinusotomy not enough?}

E Hoskison, M Daniel, N S Jones, A Sama

From Nottingham University Hospital, UK

\section{Objective}

Few patients require frontal sinus surgery. The majority of patients with frontal symptoms have tension-type headache, and whilst many patients with moderate or severe polyposis have retained secretions, these rarely cause symptoms or require surgery. Acute infection is often a 'one-off' event, and trephining can be performed if antibiotics fail. Patient selection is the key, as it is all too easy to cause stenosis of the frontal recess. In the minority who have symptoms, supported by endoscopic and/or computed tomography evidence of disease, and who do not respond to medical treatment, frontal sinusotomy may be sufficient. A subset of patients requires more complex surgery. The aim of this study was to characterise those cases in which more extensive surgery is required. 


\section{Method}

Prospective data collection on all patients undergoing frontal sinus surgery at our institution, between 30 May 1990 and 26 September 2007.

\section{Results}

Two hundred and forty-four patients underwent 275 endoscopic frontal sinus procedures. The mean patient age was 46.2 years (range, seven to 81 years); 91 (37 per cent) patients were female and 153 (63 per cent) were male. Overall, Draf I procedures accounted for 93.1 per cent of surgery, Draf II for 3.6 per cent and Draf III for 3.3 per cent.

The majority of endoscopic procedures (82.5 per cent; $n=227$ ) were performed on the frontal sinus as the first procedure; of these, 94.3 per cent were Draf I, 4.0 per cent Draf II and 1.8 per cent Draf III. This fell to 13.5 per cent for the second procedure, 3.3 per cent for the third procedure and 0.4 per cent for the fourth and fifth procedures.

Of the 256 Draf I procedures, 128 were for nasal polyposis, 48 for infective sinusitis, 39 for mucocele, 35 for inverted papilloma and six for other conditions (including spindle cell tumour and trauma). Of the 10 Draf II procedures, two were for nasal polyposis, one for infective sinusitis, one for inverted papilloma, three for mucocele and three for other diagnoses. Of the nine Draf III procedures, three were for nasal polyposis, one for inverted papilloma, four for mucocele and one for spindle cell tumour. Twenty per cent of procedures for inverted papilloma required more extensive surgery than a Draf I procedure, whereas for spindle cell tumours this procedure was inadequate.

\section{Conclusions}

The Draf I procedure is usually indicated in primary surgery, especially if the diagnosis is nasal polyposis. In contrast, the Draf II and III procedures are required in cases of mucocele and neoplasm.

\section{Functional endoscopic dilatation of sinuses: preliminary findings of a comparative trial}

P Achar, N Kumar, S Duvvi, I Mitra

From the Wrightington, Wigan \& Leigh NHS Trust, Wigan, UK

\section{Objective}

To compare the effectiveness and safety of functional endoscopic dilatation of sinuses with that of functional endoscopic sinus surgery (FESS), in patients with chronic rhinosinusitis who have failed medical therapy.

It is now well understood that normal physiological sinus functioning depends on the patency of ostiomeatal units, normal mucociliary function, and normal quantity and quality of secretions. Over the past two decades, the surgical management of rhinosinusitis has completely changed. This is due to technical advances in endoscopic systems, and to the recognition of the importance to normal sinus function of mucociliary flow and ventilation through the anatomical ostia.

Innovative catheter-based technology has improved treatment of several conditions such as coronary artery disease, peripheral vascular disease and stroke. Recently, catheter devices have been developed for the paranasal sinuses. Functional endoscopic sinus dilatation is a new technique of sinus ostial balloon dilatation which is specifically aimed at restoring the patency of the sinuses without removing any tissue, thereby decreasing morbidity. This procedure is even more minimally invasive than conventional FESS.

\section{Method}

Ethical committee approval was obtained for a comparative trial. We recruited 20 patients with signs and symptoms of chronic rhinosinusitis refractory to medical treatment who were proceeding to surgery. These patients underwent either functional endoscopic dilatation of sinuses or FESS. They also completed a Sino-Nasal Outcome Test (SNOT) 20 questionnaire and underwent a saccharine test prior to and three months after the intervention.

\section{Results}

The Sino-Nasal Outcome Test (SNOT) 20 scores for both patient groups showed similar, consistent symptomatic improvement compared with baseline measures. The saccharine time was reduced in both groups. No significant post-operative complications were recorded in either group. None of the functional endoscopic sinus dilatation group had any bleeding, and they were ready for discharge within hours of the procedure.

\section{Conclusions}

Functional endoscopic dilatation of sinuses appears to be as safe and effective as FESS in relieving ostial obstruction, and, in addition, has the potential to be undertaken as a day case procedure. Preliminary results from our UK trial showed that patients had a positive outcome and a pleasant post-operative experience. 